\title{
Monitoring the dynamics of wheat stem elongation: genotypes differ at critical stages
}

\author{
Lukas Kronenberg $(\mathbb{D} \cdot$ Kang Yu $\cdot$ Achim Walter $\cdot$ Andreas Hund
}

Received: 6 December 2016/ Accepted: 23 June 2017 / Published online: 4 July 2017

(C) The Author(s) 2017. This article is an open access publication

\begin{abstract}
Stem elongation is a critical phase for yield formation in wheat (Triticum aestivum). This study proposes the use of terrestrial laser scanning (TLS) for phenotyping of growth dynamics during wheat stem elongation in high temporal resolution and high throughput in the field. TLS was implemented on a novel field phenotyping platform carrying a cable suspended sensor head moveable in 3D over a 1 ha field. Canopy height was recorded on 335 winter wheat genotypes across two consecutive years. Scans were done in 3-d intervals during the stem elongation phase. Per day, 714 plots (two replications plus checks) were scanned within $3.5 \mathrm{~h}$. The results showed that canopy height increased linearly with thermal time. Based on this linearity,
\end{abstract}

This article is part of the Topical Collection on Plant Breeding: the Art of Bringing Science to Life. Highlights of the 20th EUCARPIA General Congress, Zurich, Switzerland, 29 August-1 September 2016.

Edited by Roland Kölliker, Richard G. F. Visser, Achim Walter \& Beat Boller.

Electronic supplementary material The online version of this article (doi:10.1007/s10681-017-1940-2) contains supplementary material, which is available to authorized users.

L. Kronenberg $(\square) \cdot$ K. Yu $\cdot$ A. Walter $\cdot$ A. Hund Institute of Agricultural Sciences, Group of Crop Sciences, ETH Zürich, Universitätstrasse 2, LFW C54.1, 8092 Zurich, Switzerland

e-mail: lukas.kronenberg@usys.ethz.ch
15 and $95 \%$ of final height were used as proxy measures for the onset and termination of stem elongation, respectively. We observed high heritability between 0.76 and 0.91 for the onset, termination and duration of stem elongation. The onset of stem elongation showed a positive covariance with the termination of stem elongation and final height indicating some regulatory dependencies. Yet there was no apparent relationship between onset and duration of stem elongation. Due to its precision, the TLS method allows to measure the dynamics of stem elongation in large sets of genotypes. This in turn offers opportunities to investigate the genetic control of the transitions between early vegetative growth, stem elongation and flowering. Understanding the genetic control of these transitions is an important milestone towards knowledge-based crop improvement.

Keywords Wheat - Stem elongation - Growth · Phenotyping $\cdot$ Development

\section{Introduction}

Stem elongation (SE) is a critical phase for yield formation in wheat (Triticum aestivum) and has been proposed as a target site to improve yield and environmental adaptation of wheat (Miralles et al. 2000; González et al. 2003). 
Physiological background

Although SE itself is a growth trait, it has been shown to be strongly related to developmental progress in the field (Slafer et al. 1995). Under field conditions, SE starts after the formation of the terminal spikelet and ends with anthesis (Slafer et al. 2009). Between these two events, active spike growth takes place (Slafer et al. 2009). Due to this co-occurrence, the SE phase is considered to be critical for potential yield in wheat. This has been proposed quite early (Hudson 1934; Fischer 1985; Slafer et al. 2001) and was widely confirmed in later years (Kirby 1988; Slafer et al. 1996, 2001). Spike dry weight at anthesis is largely related to grain number per spike (Fischer 1985; Slafer et al. 2001), which in turn has been found to be more yield determining than individual grain weight (Slafer et al. 2001 and references cited therein), especially in connection with environmental variation of yield (Fischer 1985; Calviño and Monzon 2009).

The number of fertile florets and the related spike dry weight depend on the duration of the SE and concurrent spike growth period (Slafer et al. 1996, 2001; González et al. 2003). It has been proposed that a prolonged SE phase would allow for more dry matter accumulation by the spike due to its longer growth phase, which would ultimately increase yield (Slafer et al. 1996; Miralles and Slafer 2007; Whitechurch et al. 2007).

Numerous studies focus on photoperiod effects on SE duration (Slafer et al. 2001; Whitechurch and Slafer 2002; González et al. 2003; Miralles et al. 2003; Fischer 2011). It was shown under controlled (Miralles et al. 2000) as well as under field conditions (Whitechurch and Slafer 2002; González et al. 2003), that manipulation of photoperiod affects SE duration. The genetics of photoperiod response in wheat have been studied and associated alleles have been found (Mohler et al. 2004; Slafer et al. 2009). Yet, many uncertainties remain in understanding the genetic control of SE duration (Fischer 2011).

The investigation of growth dynamics during stem elongation is a promising approach to gain further insight in the physiological and genetic mechanisms that control stem elongation duration and its effect on yield. However, studies on wheat growth and development during stem elongation are usually based on a relatively small number of genotypes (Porter and Gawith 1999). A precise characterisation of the growth dynamics during stem elongation along with the identification of potential key stages during this period in a large set of genotypes could therefore provide helpful information.

Growth dynamics during stem elongation have been studied with focus on single internodes or leaves (Kirby 1988). Internode elongation has been described as a sigmoidal curve with a prominent middle phase of constant growth in wheat (Kirby 1988) as well as in maize (Fournier and Andrieu 2000). On canopy level over the whole SE period, height growth can therefore be interpreted as multiple stacked sigmoidal curves. We assume that this may be well explained by a linear function.

Growth phenotyping

The quantification of SE growth dynamics and their response to environmental variables requires accurate plant height measurements in high temporal resolution. Especially if the aim is to perform genetic studies, these measurements have to be done on a large number of genotypes at the same time. Traditional methods (i.e., measurement by yardstick) lack the necessary throughput and accuracy to perform this task.

In recent years, many novel measurement techniques have become available from the field of plant phenotyping, using a wide range of optical sensors (see Walter et al. 2015 for a review). 3D-Laserscanning has been established as a tool to capture plant architecture under lab- or greenhouse conditions (e.g. Dornbusch et al. 2012; Paulus et al. 2014; Kjaer and Ottosen 2015). Recently, field phenotyping for height development has been achieved in several platforms (Virlet et al. 2016). 3D terrestrial laser scanning (TLS) has successfully been used for wheat, maize and soybean canopy height measurements in the field (Friedli et al. 2016). The method developed by Friedli et al. (2016) involves a laser scanner mounted on a tripod. Multiple scans at different positions in the observed field, registered by the means of stationary reference targets, resulted in accurate canopy height measurements under high temporal resolution (Friedli et al. 2016). The scanner has a technical accuracy of $0.2 \mathrm{~cm}$ in $10 \mathrm{~m}$ distance. The accuracy of the system measured as average position deviations of the stationary reference targets over time was $0.84 \mathrm{~cm}$ with a standard deviation of $0.39 \mathrm{~cm}$. Linear 
regression between hand measured canopy height and TLS canopy height yielded an $\mathrm{R}^{2}$ of 0.99 (Friedli et al. 2016).

Very recently, a unique multi-sensor field phenotyping platform (FIP) allowing for non-invasive measurements on an area of 1 ha by means of a cable-suspended sensor head was developed and built at ETH Zürich (Kirchgessner et al. 2016). The TLS canopy height measurement approach developed by Friedli et al. (2016) was integrated in the system and successfully tested for soybean height measurements (Kirchgessner et al. 2016).

In the present study, TLS plant height measurement under field conditions with the FIP is applied in a set of 335 European wheat genotypes. The set comprises genotypes from the GABI Wheat association panel which consists of European elite cultivars (Kollers et al. 2013) complemented with Swiss wheat varieties. The aims of this study are to:

(A) Test the capability of the measurement system for accurate wheat canopy height measurements in high temporal resolution.

(B) Determine growth dynamics of European wheat during stem elongation.

(C) Evaluate the hypothesis that there is considerable genetic diversity at critical stages of this dynamics.

(D) Determine interactions between the different stages and their relationship to final plant height.

\section{Material and methods}

Plant material and experimental design

During the wheat seasons 2015 and 2016 a field experiment was conducted at the research station of the institute of agricultural science of ETH in Eschikon 33, 8315 Lindau, Switzerland $\left(47.449^{\circ} \mathrm{N}\right.$, $8.682^{\circ} \mathrm{E}, 520 \mathrm{~m}$ a.s.l.; soil type: eutric cambisol). In 2015, a total of 339 European winter wheat cultivars comprising 300 varieties from the GABI Wheat panel (Kollers et al. 2013) complemented with important Swiss cultivars were sown in an augmented design consisting of two lots. Each was divided into 17 rows and 21 columns resulting in 357 micro-plots with a size of 1.7 by $1.125 \mathrm{~m}$. One variety was repeated 19 times per lot in order to account for field heterogeneity (see Fig. 1b). Border effects were minimized by surrounding the lots with buffer plots. Sowing was done on October 20, 2014 with a drill sowing machine at a blade distance of $0.125 \mathrm{~m}$ resulting in 400 plants $\mathrm{m}^{-2}$. Fertilization and plant protection was performed according to recommended agricultural practice. In 2016, the experiment was repeated with slight alterations to the design. 335 genotypes were sown in two lots with 18 rows and 20 columns each. Four old Swiss varieties present in the 2015 field experiment were excluded due to their lodging susceptibility. The sowing date for the 2016 season was October 13, 2015.

Canopy height measurements

Canopy height was measured every 3-4 days from April 16 until July 10, 2015 and from April 11 until July 42016 by using a FARO ${ }^{\circledR}$ Laser Scanner Focus3D S 120 (Faro Technologies Inc., Lake Mary USA) mounted on the FIP (Fig. 1a, for a detailed description of the system see Kirchgessner et al. 2016). The laser scanner produces a three dimensional point cloud representing the scanned area relative to the scanner. In each lot, 16 scans were taken at defined positions on every measurement date. The 16 resulting scan point clouds were then stationed and merged together into one single scan point cloud using the software Scene 5.4.4.41689 (Faro Technologies Inc., Lake Mary USA). The stationing of the scans was facilitated by the use of eight spherical reference targets with a diameter of $20 \mathrm{~cm}$ (Laserscanning Europe GmbH., Magdeburg Germany), positioned on fixed locations in the field. For a detailed description of the scanner and the applied settings, see Friedli et al. (2016).

Average canopy height of each plot was extracted from the scan point cloud using the custom MATLAB (version 2013b, The MathWorks Inc., Natick, MA, 2000) application CAHST. The software including a manual and example data is available for download from SourceForge (http://sourceforge.net/projects/ cahst4tls). In a first step, the z-coordinates (i.e., the height) of all scan points were corrected by the soil level by subtracting the respective z-coordinates of a height map of the soil. The soil map was retrieved from a scan of the soil performed 6 weeks after sowing when soil was still largely visible. This resulted in a height map for the complete scan point cloud for every 

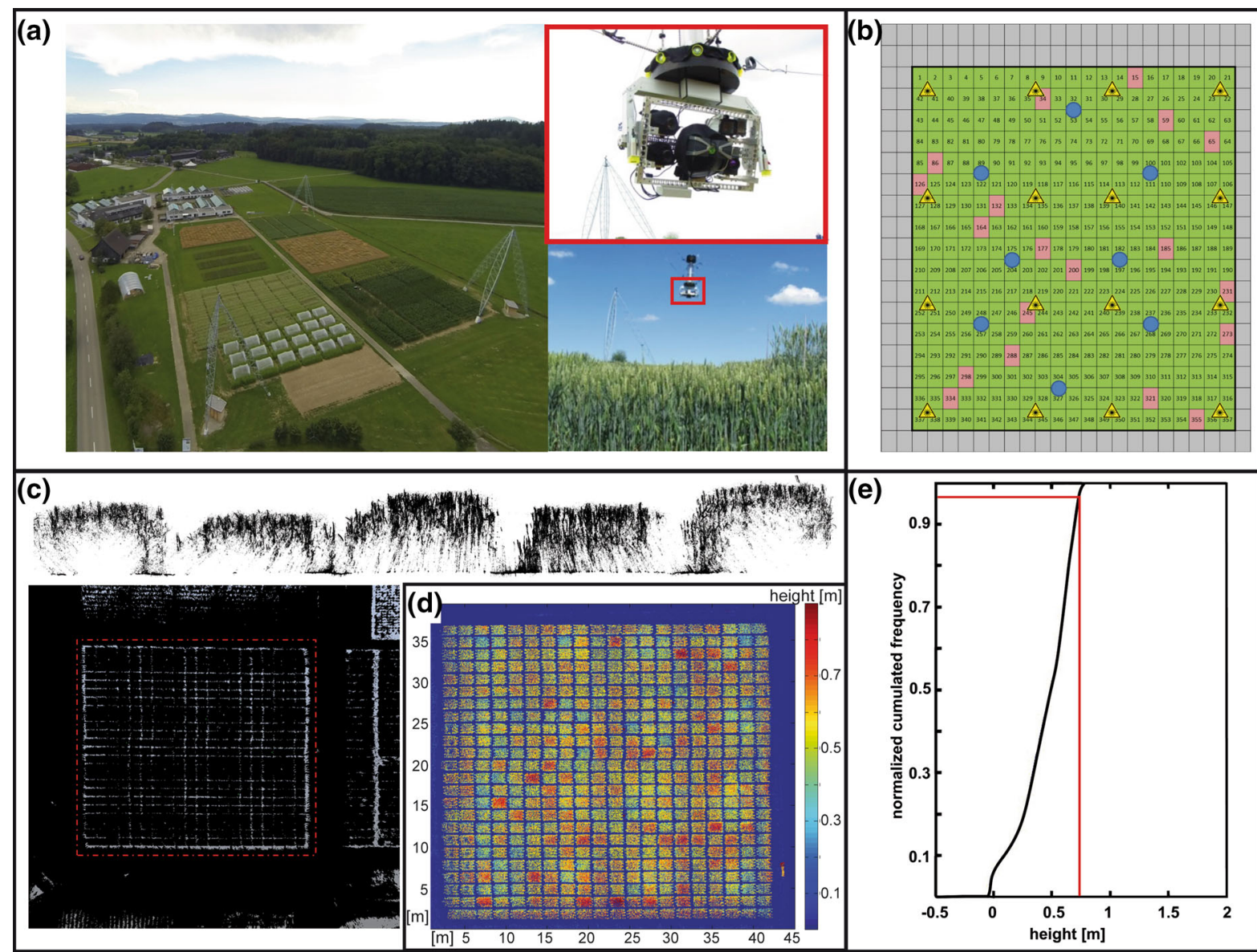

(e)

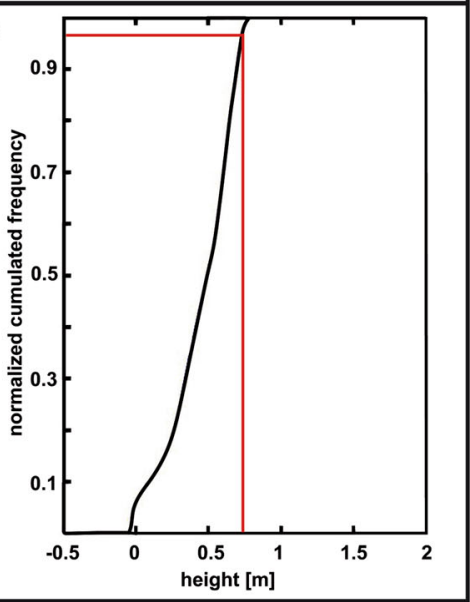

Fig. 1 Overview of the Field Phenotypig Platform (FIP) and the 3D terrestrial laser scanning (TLS) system to measure plant height: a FIP overview with poles sustaining the rigging system (picture supplied by D. Constantin, M. Rehak and Y. Akhtman, EPFL ENAC TOPO) and sensor head; b positions of the TLS (triangles), reference sphere (dots) and "check" varieties (red squares); c scan point cloud with highlighted area of the experimental field and a cross section through the scan point cloud region of five adjacent plots; d height map of soil corrected point cloud a scan of one lot; e normalized cumulated frequency of the height distribution of scan points for one plot; the red line shows the 97 th percentile. (Color figure online) measurement date (Fig. 1d). In a second step, the point height was extracted for the centre $(0.5$ by $0.5 \mathrm{~m})$ of each plot by applying a grid. Third, the normalized cumulated point height frequency was calculated. Of this, the 97th percentile was taken as canopy height of the plot in question (Fig. 1e). Friedli et al. (2016) tested several filtering approaches to extract canopy height from scan point clouds. They found the 99th percentile of all points to be the best measure for canopy height in wheat due to the exclusion of outliers and therefore reduced risk of overestimation. In our data however, percentiles above the 97th percentile didn't exclude critical outliers. All further data analysis was performed using the software RStudio (RStudio Team 2015) with the environment R (R Core Team 2015).

In a subset of 48 plots per lot, plant height was measured manually with a commercial yardstick in 2015. On every measurement day the average height of ten randomly chosen plants in the centre of each plot was regarded as plot canopy height. For the 96 plots in which reference measurements were conducted, TLS plant height was correlated with manual plant height. To test the accuracy of the TLS measurement, a linear model fit was applied over all measurement dates. 
Meteorological data

Temperature data was retrieved from a weather station of the federal Swiss meteorological network Agrometeo (www.agrometeo.ch) based in Lindau at ca. $250 \mathrm{~m}$ distance to the field trial. Hourly maximum and minimum temperature was recorded $5 \mathrm{~cm}$ above soil. Based on this data, growing degree-days (GDD; McMaster and Wilhelm 1997) were calculated for both seasons following:

Tmean $_{d}=\frac{\sum \frac{\max _{d, h}+\min T_{d, h}}{2}-\text { baseT }}{24}$,

$G D D=\sum_{d=1}^{n}$ Tmean $_{d}$

where Tmean $_{d}$ is the mean temperature for day $d$ after sowing, $\max T_{d, h}$ and $\min T_{d, h}$ are hourly maximum and minimum temperatures for that day and baseT is the base temperature set at $0{ }^{\circ} \mathrm{C}$. In cases of $\left(\max T_{d, h}+\min T_{d, h}\right)<0$, the term was set to zero. $G D D$ are the summed up daily temperature sums from 1 to $n$ days after sowing.

There were data gaps of 4 and $5 \mathrm{~h}$ on 2015-03-27 and 2016-06-20 respectively. These were filled with temperature data measured at $10 \mathrm{~cm}$ above soil by the weather station located at the research station.

Growth dynamics

Linear regression of canopy height against GDD until final canopy height $(\mathrm{FH})$ was performed for each plot separately following:

$C H=a+b \cdot G D D+\varepsilon$

where $C H$ and $G D D$ are the canopy height and the growing degree days since sowing, respectively, on the different measurement dates and $\varepsilon$ is the residual. The estimated parameter $a$ and $b$ represent the intercept and slope. Calculation was done using $\mathrm{R}$ lmList() (Bates et al. 2015).

Final height was defined as the inflection point of the canopy height versus time curve for each plot (Fig. 2). This was done with an algorithm combining the maximum canopy height value, the mean value of the last five measurement points and the point of maximum difference between measurement points and a straight line drawn between first and last

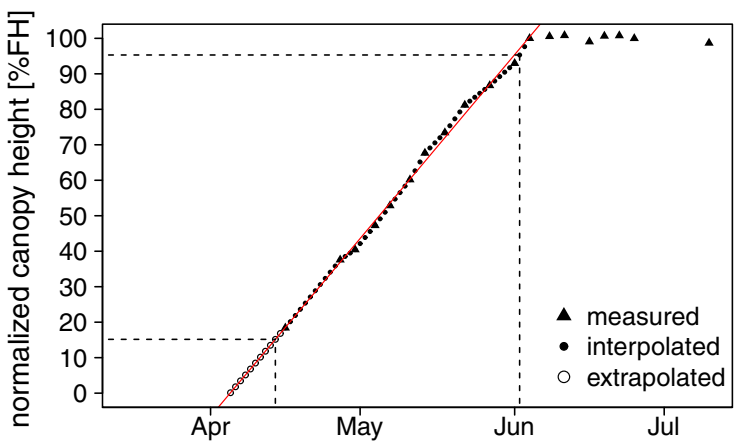

Fig. 2 Normalized canopy height versus time of a single wheat plot. Black dots show the interpolated canopy height between measurement points (black triangles). The solid red line shows the linear regression line of canopy height until final height versus time. Based on the linear regression, canopy height was extrapolated to determine the date of $15 \%$ final height (black circles). Dotted black lines show the date of $15 \%$ (left) and $95 \%$ (right) final height respectively. (Color figure online)

measured point. This method created false values e.g., in the case of lodging incidents. Therefore, for each plot FH was visually checked on plotted canopy height versus time including date and final height calculated by the algorithm and manually corrected in case of error. In addition, GDD was calculated from sowing until $15 \% \mathrm{FH}\left(\mathrm{GDD}_{15}\right)$ and $95 \% \mathrm{FH} \mathrm{GDD}_{95}$ were reached. This was done by linear interpolation of normalized plant height between consecutive measurement dates. Some genotypes already exceeded $15 \% \mathrm{FH}$ at the first measurement date. In those cases, $\mathrm{GDD}_{15}$ was obtained by linear extrapolation of normalized plant height to days prior to the first measurement date, based on the linear regression slope of measured plant height until FH versus time (Fig. 2). SE duration $\left(\mathrm{GDD}_{\mathrm{SE}}\right)$ was calculated as the difference between $\mathrm{GDD}_{95}$ and $\mathrm{GDD}_{15}$. The five parameters $\mathrm{FH}$, intercept of canopy height versus GDD, GDD 15 , $\mathrm{GDD}_{95}$ and $\mathrm{GDD}_{\mathrm{SE}}$ were used to quantify genotype specific growth habits.

Genotypic predictors across both years were calculated in a two-step approach using the R-package asreml (Butler 2009). In a first step, a linear mixed model was applied for each individual year:

$\mathrm{Y}_{\mathrm{ijkl}}=\mu+\mathrm{g}_{\mathrm{i}}+\mathrm{L}_{\mathrm{j}}+\mathrm{LR}_{\mathrm{jk}}+\mathrm{LC}_{\mathrm{jl}}+\varepsilon_{\mathrm{ijkl}}$,

where $\mathrm{Y}_{\mathrm{ij}}$ is either $\mathrm{GDD}_{15}, \mathrm{GDD}_{95}, \mathrm{GDD}_{\mathrm{SE}}, \mathrm{FH}$ or the slope of canopy height versus time, $\mu$ is the respective intercept, $\mathrm{g}_{\mathrm{i}}$ is the effect of the ith genotype $(\mathrm{i}=1, \ldots$, $335), L$ is the $j$ th replicate $(j=1,2$ for replicate in 
each different lot of the FIP) and $\varepsilon_{\mathrm{i}, \mathrm{j}}$ is the residual error. To control spatial variability additional incomplete blocks were defined where LR is the effect of the kth row in the jth lot and LC is the effect of the lth range in the jth lot. A first order autocorrelation (AR1) model was applied for each lot to account for rangeand row wise autocorrelation among plots.

In a second step, a linear model was applied on the genotypic best linear unbiased estimates (BLUEs) from both individual years to obtain genotypic best linear unbiased predictors (BLUPs) across both years:

$\mathrm{Y}_{\mathrm{ij}}=\mu+\mathrm{g}_{\mathrm{i}}+\mathrm{y}_{\mathrm{j}}+\varepsilon_{\mathrm{i}, \mathrm{j}}$

where $\mathrm{Y}_{\mathrm{ij}}$ is either $\mathrm{GDD}_{15}, \mathrm{GDD}_{95}, \mathrm{GDD}_{\mathrm{SE}}, \mathrm{FH}$ or the slope of canopy height versus time, $\mu$ is the intercept $y_{j}$ the fixed effect of the jth year $\left(\mathrm{j}=2014\right.$ and 2015), $\mathrm{g}_{\mathrm{i}}$ is the random effect ith genotype; and $\varepsilon_{\mathrm{ij}}$ the residual error including the genotype-by-year interaction.

For all traits, heritability was calculated from genotypic mean values following (Eq. 6):

$\mathrm{h}^{2}=\frac{\sigma_{G}^{2}}{\sigma_{G}^{2}+\frac{\sigma_{\varepsilon}^{2}}{\mathrm{R}}}$

where $\sigma_{G}^{2}$ and $\sigma_{\varepsilon}^{2}$ are the genetic and the error variance, respectively and $\mathrm{R}=2$ is the number of replicates (years) per genotype (Falconer and Mackay 1996).

The experiment is being repeated in the wheat season 2017. In order to validate findings related to the beginning of stem elongation (see results; Fig. 7), destructive samples were taken on April 07 (before stem elongation stared) and May 12, 2017 (during early stem elongation), in a subset of 14 genotypes. These comprised the seven earliest (RUNAL, QUATUOR, CARIBOU, CH COMBIN, RICHEPAIN GARCIA, CH CLARO) and seven latest genotypes (SEMPER, NUTKA, RYWALKA, OSTKA STRZELECKA, TONACJA, ZENITH, ZYTA) for GDD $_{15}$ based on best linear unbiased predictors of all 335 genotypes across both years 2015 and 2016. On both sampling dates, three plants per plot in the two replications per genotype were destructively sampled. For each genotype, the average plant size (length from stem base to leaf tip), the height of the ear relative to the stem base and the number of nodes (according to Lancashire et al. 1991) was recorded. Analysis of variance was performed on the individual sampling dates to test for differences between the early and the late group for each trait, by applying a linear mixed model with group and replicate as fixed- and genotype as random variable.

\section{Results}

Verification of TLS plant height measurements

Linear regression between TLS and manual plant height over all measurement dates showed a very strong linear relationship, with $\mathrm{R}^{2}=0.99$, slope $=1.02$ and intercept $=-0.096$ (Fig. 3). Pearson correlation coefficients for individual measurement dates varied between 0.80 and 0.98 . Plant height measurements were consistent over time.

\section{Growth dynamics}

The average canopy height of all 335 genotypes observed in this study showed similar development in both growing seasons 2015 and 2016 (Fig. 4). Canopy height development clearly followed a linear trend across the whole SE period. The 2 years differed mainly in the intercept of canopy height versus time with only marginal differences in slopes. Temperature sums across winter and spring (meteorological seasons) were similar in both years, with 1080 GDD in 2015 and 1074 GDD in 2016. However, there were considerable differences when single seasons were compared: The winter season 2014/2015 was colder compared to the winter 2015/2016, with 145 GDD

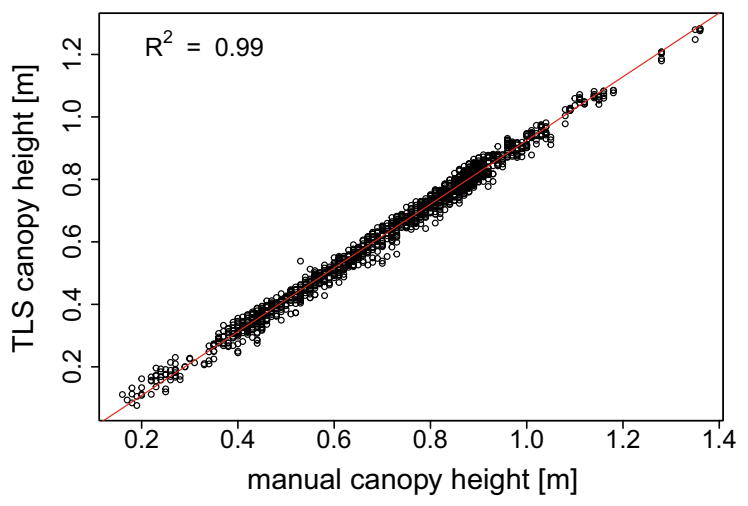

Fig. 3 Linear regression between canopy height measured with terrestrial laser scanning and manually measured canopy height. Data is based on a subset of 96 plots measured on 18 consecutive measurement dates. (Color figure online) 


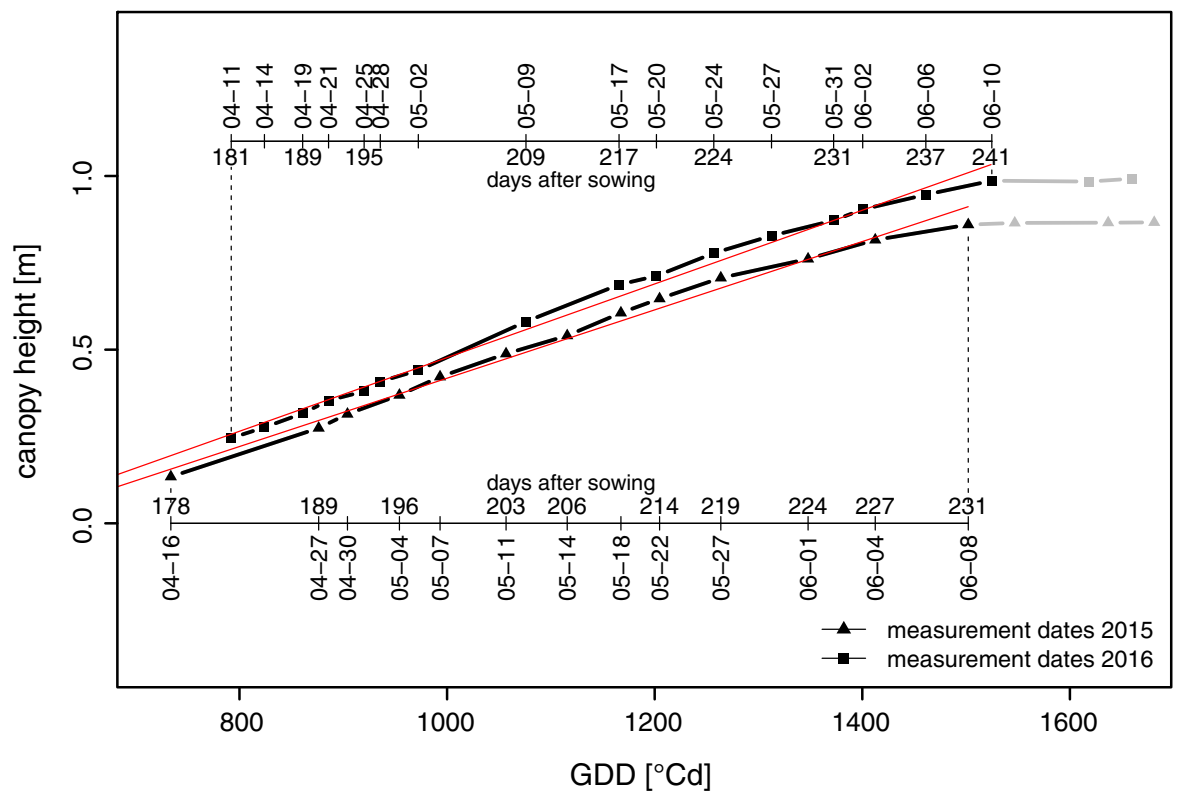

Fig. 4 Average canopy height of all 335 genotypes plotted versus growing degree days since sowing (GDD) for each measurement date in 2015 (triangles) and 2016 (squares). The

versus 234 GDD from December 1 until February 28/29. Spring (March 1 until May 31) on the other hand was warmer in 2015, with 935 GDD compared to 840 GDD in 2016 (Fig. 5). Average canopy height was considerably higher in 2016 compared to 2015 . Across both years, the date when average final height was reached differed only by 2 days.

Linear regression of canopy height against time and GDD for individual plots showed a strong linear relationship. Correlation between canopy height and

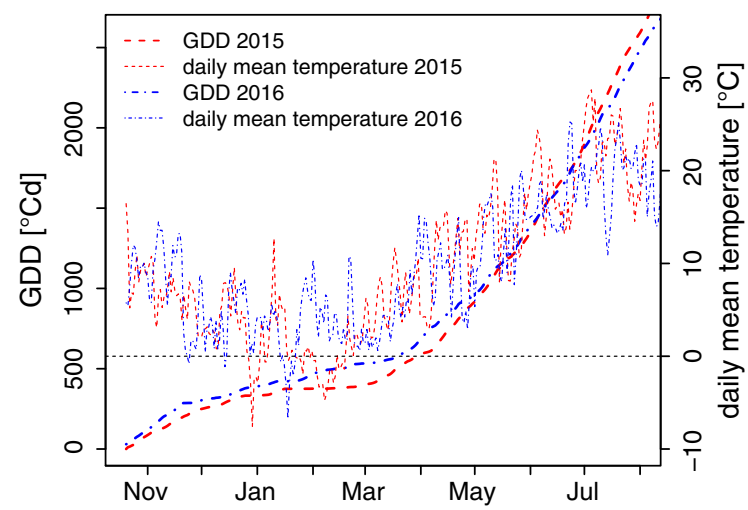

Fig. 5 Climate conditions for the two wheat growth seasons (red dashed Oct 2014-Jul 2015; blue dot-dash: Oct 2015-Jul 2016. Thick lines show growing degree days since sowing, thin lines show daily mean air temperature. (Color figure online) solid red lines show the linear regression line of average plant height versus GDD. (Color figure online)

time yielded an average $\mathrm{R}^{2}$ of 0.99 in both years, ranging from 0.92 to 0.99 in 2015 and from 0.81 to 0.99 in 2016. Canopy height versus GDD had an average $\mathrm{R}^{2}$ of 0.98 ranging from 0.92 to 0.99 in 2015 and an average $R^{2}$ of 0.99 ranging from 0.78 to 0.99 in 2016 (Fig. S1).

Analysis of variance revealed significant genotypic differences in linear height development (slopes of the linear model; Eq. 3) as well as for $\mathrm{GDD}_{15}, \mathrm{GDD}_{95}$ $\mathrm{GDD}_{\mathrm{SE}}$ and FH over 2 years $(\mathrm{p}<0.001)$. All these traits had high heritabilities across 2 years, ranging from 0.74 to 0.93 (Table 1). Best linear unbiased

Table 1 Heritabilities across both years for final height $(\mathrm{FH})$, intercept (Intercept $\mathrm{CH}_{\mathrm{CH}} \sim \mathrm{GDD}$ ) and slope (Slope $\mathrm{CH}_{\mathrm{GDD}}$ ) of linear regression between canopy height and growing degree days (GDD), GDD until 15\% $\left(\mathrm{GDD}_{15}\right)$ and $95 \%\left(\mathrm{GDD}_{95}\right)$ final canopy height and duration of stem elongation $\left(\mathrm{GDD}_{\mathrm{SE}}\right)$

\begin{tabular}{ll}
\hline Trait & Heritability \\
\hline FH & 0.96 \\
Intercept $_{\mathrm{CH} \sim \text { GDD }}$ & 0.88 \\
Slope $_{\mathrm{CH} \sim \mathrm{GDD}}$ & 0.93 \\
$\mathrm{GDD}_{95}$ & 0.91 \\
$\mathrm{GDD}_{15}$ & 0.85 \\
$\mathrm{GDD}_{\mathrm{SE}}$ & 0.76 \\
\hline
\end{tabular}


predictors (BLUPs) for the genotype effects showed high variability, with a maximum difference of 159 GDD for $\mathrm{GDD}_{15}, 298 \mathrm{GDD}$ for $\mathrm{GDD}_{95}, 224 \mathrm{GDD}$ for $\mathrm{GDD}_{\mathrm{SE}}$ and $0.59 \mathrm{~m}$ for FH (Fig. S2). The distributions of BLUPs showed negative skewness for $\mathrm{GDD}_{15}$ and $\mathrm{GDD}_{95}$ and positive skewness for $\mathrm{GDD}_{\mathrm{SE}}$ and FH, indicating a slight tendency for short, late genotypes with short stem elongation periods in the population.

Correlation analysis among the traits used to characterize genotypic growth habit showed strong to very strong positive correlations among $\mathrm{FH}, \mathrm{GDD}_{15}$ and $\mathrm{GDD}_{95}$ (Fig. 6). There was a high correlation between $\mathrm{GDD}_{15}$ and $\mathrm{GDD}_{95}$ and between $\mathrm{GDD}_{95}$ and $\mathrm{GDD}_{\mathrm{SE}}$. In contrast, $\mathrm{GDD}_{15}$ versus $\mathrm{GDD}_{\mathrm{SE}}$ showed a very weak negative correlation $(r=-0.09)$. The slope of the linear regression correlated strongly with $\mathrm{FH}$ and $\mathrm{GDD}_{15}$ and weakly with $\mathrm{GDD}_{95}$.

Correlations among $\mathrm{GDD}_{15}, \mathrm{GDD}_{95}$ and $\mathrm{GDD}_{\mathrm{SE}}$ duration suggested that these growth stages are partially inter-dependent. There were moderate correlations among $\mathrm{GDD}_{15}, \mathrm{GDD}_{95}$ and final plant height. Stem elongation duration was strongly linked with $\mathrm{GDD}_{95}$ but not to any other observed trait. The interdependencies among traits suggest that they are not independently selectable within the evaluated set of genotypes. The response of $\mathrm{GDD}_{95}$ and $\mathrm{FH}$ to a selection for $\mathrm{GDD}_{15}$ is illustrated in Fig. 7. The presented values for the 5th and 95th percentile are based on the average of all genotypes with $\mathrm{GDD}_{15}$ outside the 5 th percentile $(n=13)$ and outside the 95 th percentile $(n=11)$, respectively. The values for the 25th and 75th percentile are based on the average of all genotypes with values within the 5\% range around the respective percentiles $(n=14$ for the 25 th, and $n=17$ for the 75th percentile) and the values for the 50th percentile are based on the population average. Genotypes with a successively later start of stem elongation $\left(\mathrm{GDD}_{15}\right)$ reach their final height successively later and become taller. Selecting genotypes with values around the 25th and 75 th percentile of $\mathrm{GDD}_{15}$ results in variability in $\mathrm{GDD}_{95}$ and $\mathrm{FH}$ inside the range of variation explained by their covariation with GDD15 (Fig. 7, yellow rectangle). Selection in the population extremes ( $<5$ and $>95 \%$; Fig. 7 ) however exceed that range. The selection of the latest 5\% (>95\%) among the varieties for $\mathrm{GDD}_{15}$, led to the co-selection of a late final height and a tall canopy. In tendency, shorter genotypes are also earlier (positive correlation between $\mathrm{GDD}_{15}$ and $\mathrm{FH}, \mathrm{R}^{2} \sim 0.25$ ).

The seven earliest and latest genotypes for $\mathrm{GDD}_{15}$ were evaluated for morphological differences in 2017. On the first sampling date, the apical meristem was still not more than $1 \mathrm{~cm}$ above the shoot base for both groups. However, the early genotypes had on average
Fig. 6 Correlations among the traits final canopy height (FH), linear model slope of canopy height versus growing degree days (GDD; $\left.\operatorname{slp}_{\mathrm{CH} \sim \mathrm{GDD}}\right)$, GDD until $15 \%$ final height $\left(\mathrm{GDD}_{15}\right)$, GDD until 95\% final height $\left(\mathrm{GDD}_{95}\right)$, and duration of stem elongation phase $\left(\mathrm{GDD}_{\mathrm{SE}}\right)$. The upper panel shows Pearson correlation coefficients based on best linear unbiased predictors of all 335 genotypes and corresponding $\mathrm{p}$-values. The lower panel shows scatterplots and lowess curves of the value pairs between the respective traits. (Color figure online)

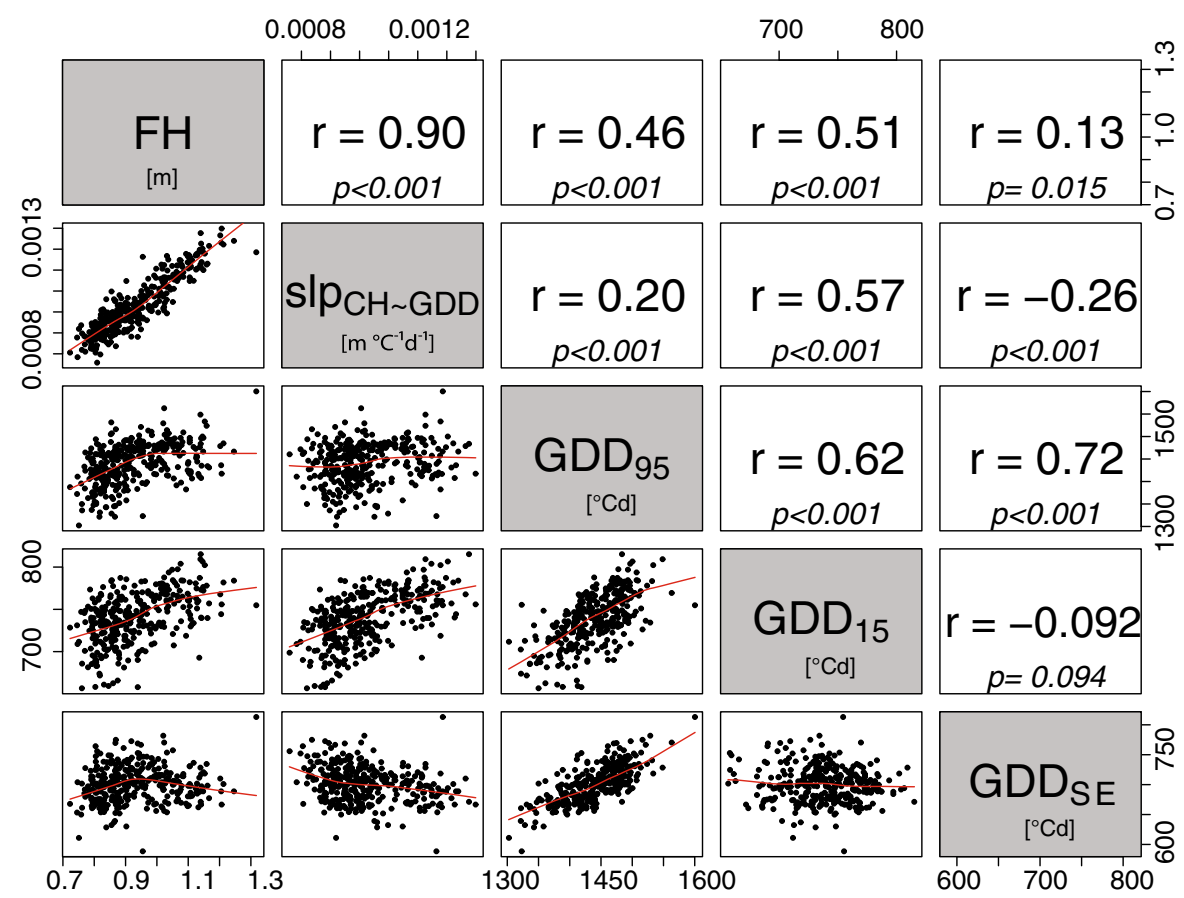




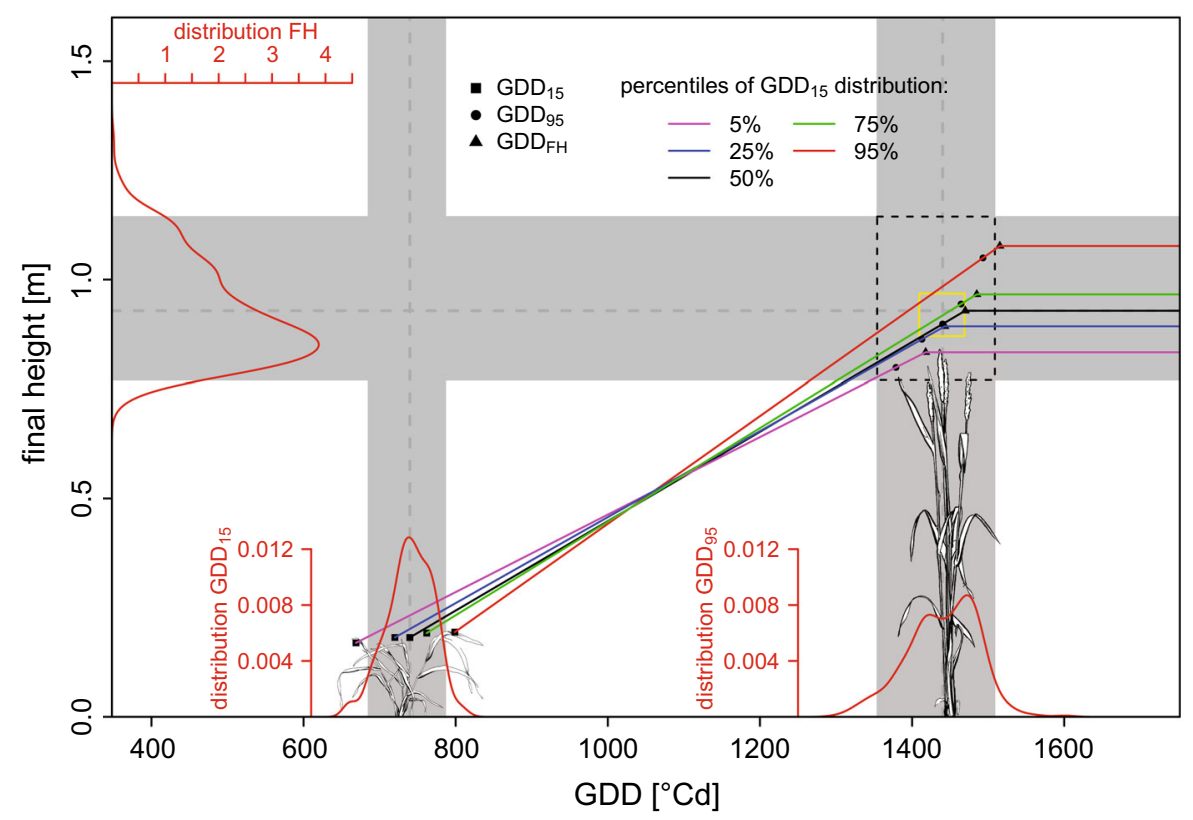

Fig. 7 The interdependency among $\mathrm{GDD}_{15}, \mathrm{GDD}_{95}$ and FH from the perspective of a selection based on $\mathrm{GDD}_{15}$. The grey shaded areas show the range of the $95 \%$ interval of the genotypic variation (BLUPs) for $\mathrm{GDD}_{15}, \mathrm{GDD}_{95}$ and FH. The width and height of the yellow rectangle shows the variance in this range of $\mathrm{GDD}_{95}$ and $\mathrm{FH}$ respectively, which is explained by

already a $5.7 \mathrm{~cm}$ greater plant size $(\mathrm{p}<0.01)$ and a slight tendency towards a greater ear height (n.s.; Fig. 8). On the second sampling date, the early genotypes had a $4.5 \mathrm{~cm}$ greater plant size $(\mathrm{p}<0.001)$, a $2.8 \mathrm{~cm}$ greater ear height $(\mathrm{p}<0.1)$ and on average 0.5 more nodes nodes $(\mathrm{p}<0.05)$ (Fig. 8).

\section{Discussion}

TLS enables high throughput phenotyping of canopy height with high spatial and temporal resolution

TLS measurements with the scanner mounted on the FIP resulted in highly precise canopy height measurements. The obtained $\mathrm{R}^{2}$ from linear regression against reference measurements are analogous to those obtained by Friedli et al. (2016). The intercept of the regression indicates an underestimation of plant height measured by TLS relative to the hand measurements by roughly $10 \%$. The explanation for this is that choosing the 97 th height percentile of the scan point
$\mathrm{GDD}_{15}$ based on the $\mathrm{R}^{2}$ of the correlation analysis (Fig. 6). Coloured lines indicated the co-selection of $\mathrm{GDD}_{95}$ and $\mathrm{FH}$ in case of a selection of genotypes of the 5th, 25th, 50th, 75th and 95th percentile (see text for details) of the genotypic variation in $\mathrm{GDD}_{15}$, respectively. (Color figure online)

cloud might be too restrictive. Taking the 100th percentile as plot height reduces the intercept to close to zero but also reduces $\mathrm{R}^{2}$ drastically (data not shown). The $97 \%$ threshold was adopted to remove numerous outliers in the point cloud of the scan, due to objects above the canopy, such as parts of the sensor head, flying insects etc. Since the discrepancy between hand and TLS measurement is constant, it can easily be corrected and does not affect the ranking between genotypes.

One aim of the present study was to test, whether the employed TLS approach with the field phenotyping platform is suitable in terms of accuracy and throughput. The obtained results show that the applied TLS approach is suitable to measure growth during SE precisely enough to detect genotypic differences in SE at a high temporal resolution. Concerning throughput, the system with the applied settings allowed measuring plant height in a total of 714 plots within $3.5 \mathrm{~h}$, including the time for setting up the device and spherical targets. This is fast enough to screen a genetic mapping population (Grieder et al. 2015), as has been confirmed in this study. With the current setting, the needed time would even allow screening 

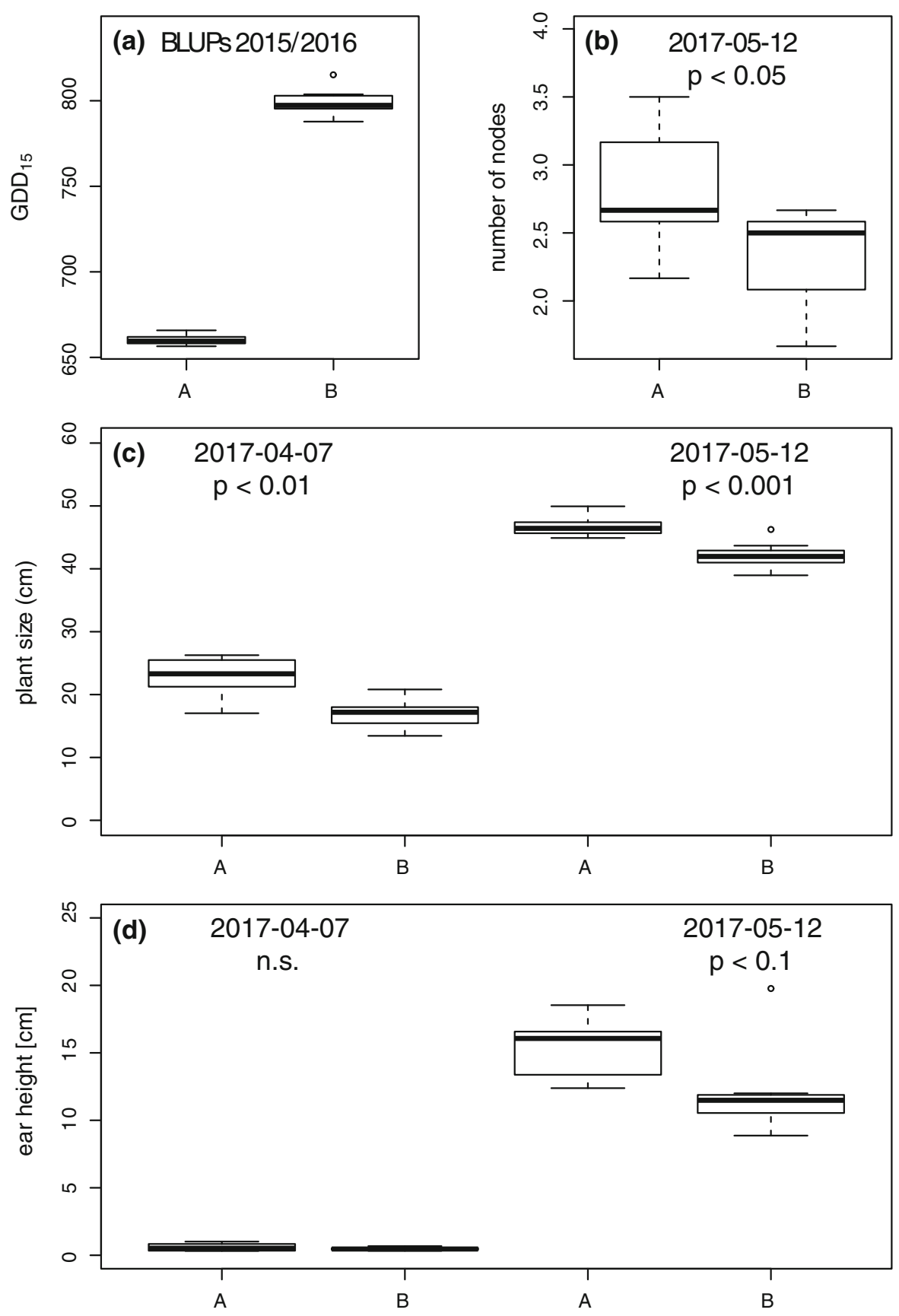

Fig. 8 Boxplots of best linear unbiased predictors (BLUPs across season 2015 and 2016) of the earliest $(A)$ and latest $(B)$ seven genotypes with regard to the growing degree days at which $15 \%$ of final plant height $\left(\mathrm{GDD}_{15}\right)$ was reached (a). Boxplots of the performance of the selected groups in 2017 with

twice a day or during the night and thus further increase temporal resolution.

In the present study, we measured wheat canopy height in a time resolution of 3 days on a large set of regard to the number of nodes at the elongating stem (b), the plant size [length from stem base to leaf tip, (c)] and ear height above the stem base (d) either before (April 7) or after the start of stem elongation (May 12). Each boxplots is based on the mean of the seven genotypes per group

genotypes which well represents European elite wheat germplasm. Previous studies investigating wheat growth during SE had a measurement frequency similar to the present study (Siddique et al. 1989; 
Flink et al. 1995) or even higher in the case of Kirby (1988) who measurement daily. The number of observed genotypes in those studies was however significantly lower compared to the present study. Kirby (1988) and Flink et al. (1995) used one variety each, the former in a greenhouse experiment, the latter in a field trial. Siddique et al. (1989) used a total of 26 varieties in three field experiments. In these studies, plant height or length of single stem organs was measured by hand. This allows for high accuracy and time resolution given a small enough number of experimental units. It would however be impossible on a scale as proposed here.

The wheat canopy showed an almost linear increase in height during the stem elongation phase

We observed a more or less linear height development for almost all genotypes between the start of stem elongation until the final height was reached. It has been reported previously, that single internode elongation in wheat (Kirby 1988) and maize (Fournier and Andrieu 2000) follows a sigmoidal trend. Based on the present study, we assume that the sum effect of the elongation dynamics of all elongating internodes can be described as linear. The results show that wheat growth during the stem elongation follows a clear linear trend. However, if growth rates between consecutive measurement dates are considered (data not shown, for an illustration see Fig. 2), consistent deviation over time from this linear trend can be observed. One explanation for this could be that the sum of sigmoidal single internode elongations does not result in a linear function. On the other hand, the linear relationship found in this study is highly significant with high $\mathrm{R}^{2}$ across all genotypes. Applying a non-linear regression did not result in a better model fit (data not shown). Therefore, SE could indeed be linear and the deviations could offer opportunities to investigate responses to short term fluctuations of environmental parameters.

TSL enabled a precise measurement of three highly heritable traits: the beginning and end of stem elongation, as well as final height

By means of TLS we could quantify three potentially independent traits: (i) the onset of stem elongation, (ii) the end of stem elongation and (iii) the final height.
Due to the linearity of stem elongation, the measuring times might be reduced in future experiments by focussing on the critical phases of the beginning and end of stem elongation. The high heritability of these traits across the 2 years indicates sufficient response to selection. Yet their co-variance indicates pleiotropic effects or epistatic interactions of the underlying genes. Such effects should be taken into account when aiming for trait-based selection. We did not take into account population structure as possible reason for these dependencies. However, the population used shows little population structure (Kollers et al. 2013) and it seems unlikely that it would be the primary cause of the observed relationship. The duration of stem elongation was primarily influenced by the variation in $\mathrm{GDD}_{95}$ while the variation in $\mathrm{GDD}_{15}$ had little influence. This independence between SE duration and the duration of prior phases has been reported before (Whitechurch et al. 2007). Our results suggest that variation in $\mathrm{GDD}_{15}$ has an indirect effect on $\mathrm{GDD}_{95}$ but does not affect SE duration.

We propose TSL measurements for determining the difficult-to-measure onset of stem elongation

The beginning of stem elongation is a stage which is usually difficult to measure non-destructively and with high throughput. Termination of SE can be regarded as a proxy measure for flowering time, which is an important breeding trait that has been much exploited (Jung and Müller 2009; Borràs-Gelonch et al. 2012). Also final height may be efficiently determined by hand measurements or, by one single TLS scan at the end of flowering. However, the variability of $\mathrm{GDD}_{15}$ (i.e., the onset of SE) could be a beneficial new proxy measure to evaluate the beginning of stem elongation. It is one of five critical stages of wheat which is frequently used in crop models (Holzkämper et al. 2015). Yet, it is the only stage among the five that is difficult to assess on a large set of genotypes. Whereas crop emergence, the 3-leaf stage, anthesis and physiological maturity can be evaluated non-destructively in a fairly rapid manner, the beginning of stem elongation must be determined destructively. Plants need to be dissected to determine the time at which the first node, which is hidden within the leaf sheaths, is approximately $1 \mathrm{~cm}$ above the tillering node (Pask et al. 2012). Accordingly, a reliable proxy measure for this critical stage will greatly enhance our 
understanding about its genetic control and importance for crop improvement.

Based on the 2 years data, we selected early and late genotypes with regard to $\mathrm{GDD}_{15}$ and evaluated them in the following season. The selected genotypes clearly differed for plant size before the onset of stem elongation. Accordingly, the selection was not only affecting timing but also growth type. As stems were not elongated at the early sampling date, the greater plant size must be related to longer leaves. This indicates, that also the baseline, i.e., the plant height before stem elongation is an important parameter, when selecting for earliness using the height dynamics. Nevertheless, the advanced earliness of the early group compared to the late group is supported by its advanced development at the second sampling date indicated by more nodes and taller plants. To what degree the two traits, taller plants with longer leaves and earlier start of stem elongation, are genetically linked needs to be subject of further evaluations. Remarkably, three of the seven earliest genotypes $(\mathrm{CH}$ CLARO, RUNAL and CH COMBIN) are elite varieties derived from the Swiss breeding program at Agroscope and widely grown in Switzerland.

\section{Conclusion and outlook}

We observed a high heritability for the onset of stem elongation. By selecting earliest and latest genotypes, we could show that the early plants were not only elongating earlier but plants were already taller. Accordingly, the initial height before stem elongation should be determined in future experiments. Large field phenotyping platforms such as the FIP are suitable for determining the genetic basis of complex traits which are difficult to assess in normal field settings. The systems stationarity confines its application to a single environment, which limits its application for breeding related studies, where multiple environments are desired. Yet the high heritabilities of the observed traits across years indicate that it might be sufficient to evaluate them in a limited number of years or environments. Furthermore, drone technology is advancing rapidly. Canopy height measurement with drones is currently possible from digital images as well as drone carried light weight laser scanners, although it might lack the accuracy as proposed in our study. However, the accuracy of UAV plant height measurement can be expected to increase in the near future. This would allow for the application of the approach proposed in this study in multiple environments. Thus, the timing of the onset and end of stem elongation could be integrated in large scale breeding programs at low labour cost. A genome wide association study (GWAS) is in progress to identify the genomic regions controlling the evaluated traits.

Acknowledgements We thank Norbert Kirchgessner for his constant help with operating the FIP and for his support during the analysis of scan point data using his software package CAHST. We also sincerely thank Hansueli Zellweger for managing and nursing our field experiment. Special thanks are dedicated to Caterina Schürch for drawing the wheat plants shown in Fig. 7 and to the ETH Crop Phenotyping class 2017 for the support during sampling and evaluation of the contrasting groups. Finally, we thank the anonymous reviewers for their helpful comments and suggestions as well as the Swiss Federal Office for Agriculture FOAG which supported this project.

Open Access This article is distributed under the terms of the Creative Commons Attribution 4.0 International License (http:// creativecommons.org/licenses/by/4.0/), which permits unrestricted use, distribution, and reproduction in any medium, provided you give appropriate credit to the original author(s) and the source, provide a link to the Creative Commons license, and indicate if changes were made.

\section{References}

Bates D, Mächler M, Bolker B, Walker S (2015) Fitting linear mixed-effects models using lme4. J Stat Softw. doi:10. 18637/jss.v067.i01

Borràs-Gelonch G, Rebetzke GJ, Richards RA, Romagosa I (2012) Genetic control of duration of pre-anthesis phases in wheat (Triticum aestivum L.) and relationships to leaf appearance, tillering, and dry matter accumulation. J Exp Bot 63:69-89

Butler D (2009) asreml: asreml() fits the linear mixed model. R package version 3.0

Calviño P, Monzon J (2009) Chapter 3-farming systems of Argentina: yield constraints and risk management. In: Sadras V, Calderini D (eds) Crop physiology. Academic Press, San Diego, pp 55-70

Dornbusch T, Lorrain S, Kuznetsov D, Fortier A, Liechti R, Xenarios I, Fankhauser C (2012) Measuring the diurnal pattern of leaf hyponasty and growth in Arabidopsis-a novel phenotyping approach using laser scanning. Funct Plant Biol 39:860-869

Falconer DS, Mackay TF (1996) Introduction to quantitative genetics. Essex, Benjamin Cummings

Fischer RA (1985) Number of kernels in wheat crops and the influence of solar radiation and temperature. J Agric Sci 105:447-461 
Fischer RA (2011) Wheat physiology: a review of recent developments. Crop Pasture Sci 62:95-114

Flink M, Pettersson R, Andrén O (1995) Growth dynamics of winter wheat in the field with daily fertilization and irrigation. J Agron Crop Sci 174:239-252

Fournier C, Andrieu B (2000) Dynamics of the elongation of internodes in maize (Zea mays L.): analysis of phases of elongation and their relationships to phytomer development. Ann Bot 86:551-563

Friedli M, Kirchgessner N, Grieder C, Liebisch F, Mannale M, Walter A (2016) Terrestrial 3D laser scanning to track the increase in canopy height of both monocot and dicot crop species under field conditions. Plant Methods 12:9

González FG, Slafer GA, Miralles DJ (2003) Floret development and spike growth as affected by photoperiod during stem elongation in wheat. Field Crops Res 81:29-38

Grieder C, Hund A, Walter A (2015) Image based phenotyping during winter: a powerful tool to assess wheat genetic variation in growth response to temperature. Funct Plant Biol 42:387-396

Holzkämper A, Fossati D, Hiltbrunner J, Fuhrer J (2015) Spatial and temporal trends in agro-climatic limitations to production potentials for grain maize and winter wheat in Switzerland. Reg Environ Change 15:109-122

Hudson PS (1934) English wheat varieties II. Development of the wheat plant. Z Züchtung 19:70-108

Jung C, Müller AE (2009) Flowering time control and applications in plant breeding. Trends Plant Sci 14:563-573

Kirby EJM (1988) Analysis of leaf, stem and ear growth in wheat from terminal spikelet stage to anthesis. Field Crops Res 18:127-140

Kirchgessner N, Liebisch F, Yu K, Pfeifer J, Friedli M, Hund A, Walter A (2016) The ETH field phenotyping platform FIP: a cable-suspended multi-sensor system. Funct Plant Biol 44(1):154-168

Kjaer KH, Ottosen C-O (2015) 3D laser triangulation for plant phenotyping in challenging environments. Sensors 15:13533-13547

Kollers S, Rodemann B, Ling J, Korzun V, Ebmeyer E, Argillier O, Hinze M, Plieske J, Kulosa D, Ganal MW et al (2013) Whole genome association mapping of fusarium head blight resistance in European Winter Wheat (Triticum aestivum L.). PLoS ONE 8:e57500

Lancashire P, Bleiholder H, Vandenboom T, Langeluddeke P, Stauss R, Weber E, Witzenberger A (1991) A uniform decimal code for growth-stages of crops and weeds. Ann Appl Biol 119:561-601

McMaster GS, Wilhelm WW (1997) Growing degree-days: one equation, two interpretations. Agric For Meteorol 87:291-300

Miralles DJ, Slafer GA (2007) Paper presented at international workshop on increasing wheat yield potential, Cimmyt, Obregon, Mexico, 20-24 March 2006 sink limitations to yield in wheat: how could it be reduced? J Agric Sci 145:139-149

Miralles DJ, Richards RA, Slafer GA (2000) Duration of the stem elongation period influences the number of fertile florets in wheat and barley. Funct Plant Biol 27:931-940

Miralles DJ, Slafer GA, Richards RA, Rawson HM (2003) Quantitative developmental response to the length of exposure to long photoperiod in wheat and barley. J Agric Sci 141:159-167

Mohler V, Lukman R, Ortiz-Islas S, William M, Worland AJ, van Beem J, Wenzel G (2004) Genetic and physical mapping of photoperiod insensitive gene Ppd-B1 in common wheat. Euphytica 138:33-40

Pask AJD, Pietragalla J, Mullan DM, Reynolds MP (2012) Physiological breeding II: a field guide to wheat phenotyping. CIMMYT, Mexico

Paulus S, Schumann H, Kuhlmann H, Léon J (2014) Highprecision laser scanning system for capturing 3D plant architecture and analysing growth of cereal plants. Biosys Eng 121:1-11

Porter JR, Gawith M (1999) Temperatures and the growth and development of wheat: a review. Eur J Agron 10:23-36

R Core Team (2015) R: a language and environment for statistical computing. R Foundation for Statistical Computing, Vienna

RStudio Team (2015) RStudio: integrated development for R. RStudio Inc, Boston

Siddique KHM, Kirby EJM, Perry MW (1989) Ear: stem ratio in old and modern wheat varieties; relationship with improvement in number of grains per ear and yield. Field Crops Res 21:59-78

Slafer GA, Halloran GM, Connor DJ (1995) Influence of photoperiod on culm length in wheat. Field Crops Res 40:95-99

Slafer GA, Calderini DF, Miralles DJ (1996) Yield components and compensations in wheat: opportunities for further increasing yield potential. In: Reynolds MP, Rajaram S, McNab A (eds) Increasing yield potential in wheat: breaking the barriers: proceedings of a workshop held in Ciudad Obregón, Sonora, Mexico. CIMMYT, Mexico, pp 101-133

Slafer GA, Abeledo LG, Miralles DJ, Gonzalez FG, Whitechurch EM (2001) Photoperiod sensitivity during stem elongation as an avenue to raise potential yield in wheat. In: Bedö Z, Láng L (eds) Developments in plant breeding. Wheat in a GLOBAL ENVIRONMENT. Springer, New York, pp 487-496

Slafer GA, Kantolic AG, Appendino ML, Miralles DJ, Savin R (2009) Chapter 12 - crop development: genetic control, environmental modulation and relevance for genetic improvement of crop yield. In: Sadras V, Calderini D (eds) Crop physiology. Academic Press, San Diego, pp 277-308

Virlet N, Sabermanesh K, Sadeghi-Tehran P, Hawkesford MJ (2016) Field Scanalyzer: an automated robotic field phenotyping platform for detailed crop monitoring. Funct Plant Biol 44(1):143-153

Walter A, Liebisch F, Hund A (2015) Plant phenotyping: from bean weighing to image analysis. Plant Methods 11:1-11

Whitechurch EM, Slafer GA (2002) Contrasting Ppd alleles in wheat: effects on sensitivity to photoperiod in different phases. Field Crops Res 73:95-105

Whitechurch EM, Slafer GA, Miralles DJ (2007) Variability in the duration of stem elongation in wheat and barley genotypes. J Agron Crop Sci 193:138-145 